\title{
2 The simple conditional analysis
}

This and the next chapter are all about learning from others. And by that I mean not just learning from their mistakes, but also learning from their insights. What I will do, therefore, is take a detailed look at the two most prevalent traditional views of abilities, extract their most appealing insights, and argue why none of them is suited to provide a comprehensive view of abilities.

The first view I will discuss, and the one that will concern us in this chapter, is what I will refer to as the "simple conditional analysis of abilities". On that view, having an ability is a matter of a certain counterfactual being true. In its most prominent version, the view states that an agent has an ability to $\phi$ if and only if the agent would $\phi$, if she so chose (Moore 1912, ch. 7). The view can be traced back to Hume, who famously held that

[b]y liberty, then, we can only mean a power of acting or not acting according to the determinations of the will; that is, if we choose to remain at rest, we may; if we choose to move, we also may. (Hume 1748, 8.23/ 95)

The simple conditional analysis used to be very popular among compatibilists in the free will debate, who, like Hume and Moore, saw it as a means to provide a compatibilist position on free will and determinism. But it was also met with sympathy by many logical positivists, including Schlick (1939) and Ayer (1946).

Different versions of the view differ with respect to the motivational state figuring in the antecedent of the counterfactual. What all of the views have in common, however, is that they analyze abilities in terms of a single counterfactual conditional, according to which the agent would have performed an action, if she had been properly motivated to perform it.

I am emphasizing that the simple conditional analysis works with just one counterfactual, because we will see in chapter 6.2 that there are more sophisticated ways of analyzing abilities by employing counterfactual conditionals. Instead of using just one counterfactual, the views we will be looking at in that section work with a large quantity of such conditionals, each of which has a highly specific antecedent. They can therefore be seen as a refined successor of the simple conditional analysis. I will subsume them under the label "the sophisticated conditional analysis" later on.

Since the sophisticated conditional analysis avoids very many of the problems that are going to be brought up for the simple conditional analysis in this chapter, I want to emphasize very clearly that this chapter deals exclusively with the simple conditional analysis and its problems. It deals with views that analyze abilities in terms of a single counterfactual conditional of the form "If

2 OpenAccess. (c) 2020 Jaster, published by De Gruyter. (cc) BY-NC-ND This work is licensed under the Creative Commons Attribution-NonCommercial-NoDerivatives 4.0 License. 
S had been properly motivated to $\phi, S$ would have $\phi$ 'ed". Seeing the limitations of that kind of view will be extremely helpful in seeing how to do better. And at this point in the book, this is just the kind of insight we are after.

For simplicity and vividness, I will concentrate on one particular version of the simple conditional analysis in what follows. And for reasons of uniformity with my own account of abilities, that version will be one which posits a counterfactual connection between the agent's intentions and her performances. More precisely, I will focus on the view that

SCAA. An agent $S$ has an ability to $\phi$ if and only if $S$ would $\phi$, if $S$ intended to $\phi$.

I would like to emphasize, however, that none of the arguments that are going to be developed in this chapter hinge on the differences between this version of the simple conditional analysis and any other version of the view. Everything I say in this chapter applies to any analysis of abilities in terms of a single counterfactual, which ties some kind of motivational state of the agent to her corresponding performances.

I think we can say without reserve that SCAA is an extremely appealing view at the outset. Abilities, we know, are modal properties - there is a difference between their instantiation and their manifestation, and the former does not require the latter. For an agent to have the ability to do a handstand, say, the agent need not actually do a handstand. Yet, of course, the ability to do a handstand clearly has something to do with the agent's effectively doing handstands; if not in the actual circumstances, then surely in possible circumstances. The conditional analysis captures this thought rather elegantly: to have the ability to do a handstand, the agent need not actually do a handstand; what she needs to do is do a handstand when intending to do one. That seems like a very reasonable requirement.

In view of the initial plausibility of the view, it is unsurprising that SCAA is still the default view in many areas of philosophy and the view many try to cling to when encountering objections. The idea that abilities will turn out to be a matter of some counterfactual or other being true is still very deeply entrenched in the contemporary debate.

In what follows, I will argue that this idea is mistaken. The claim I will establish in the course of this chapter is that abilities are not properly accounted for in terms of SCAA, no matter how exactly the counterfactual is spelled out. SCAA faces a number of structural problems, which together amount to the fail- 
ure of the view, and it does so regardless of the details of any specific version of the account. ${ }^{1}$

The structure of the section is as follows. Since we are dealing with counterfactuals a lot in this chapter, I will start, in the next section (2.1), by elaborating on the semantics of counterfactuals generally. This will provide us with a crisp terminology and a firm grip on the lines of argument to come. Readers familiar with the standard semantics for counterfactuals are well-advised to skip that section.

The subsequent sections are devoted to the critique of the simple conditional analysis. As I will argue, the analysis fails because it runs into problems with all of the adequacy conditions for a comprehensive view of abilities. The view is extensionally inadequate, because it runs into problems with masks $(\rightarrow 2.2)$ and cases in which the agent cannot form the intention to $\phi$ to begin with $(\rightarrow 2.3)$, it fails to provide an account of general and specific abilities $(\rightarrow 2.4)$, it fails to offer an account of degrees of abilities and the corresponding kind of context sensitivity that attaches to ability statements $(\rightarrow 2.5)$, and it fails to provide an understanding of non-agentive abilities $(\rightarrow 2.6)$. By the end of this chapter, it should be clear that the simple conditional analysis, while highly appealing at the outset, is deeply flawed. Abilities are not a matter of the truth of a counterfactual.

\subsection{Modal semantics: Counterfactuals}

A counterfactual, as I use the term, is a modal statement in the form of a subjunctive conditional - it states that $p$ would be the case, if $q$ were the case. This is a modal statement insofar as p, and thus q, need not be the case for such a statement to turn out true. Most counterfactuals have an antecedent which specifies a non-actual event; thus, the name "counterfactual". Despite the name, however, this is not essentially so. Some subjunctive conditionals specify an actual even in their antecedent, and they typically also go by the name "counterfactual". ${ }^{2}$ I'll stick to that practice throughout the book.

Let's get some counterfactuals before us. Here are some true ones. If I had gotten up at 6 , I would have heard the church bells; if you were the president

1 Note that the criticism I am going to develop is not meant to provide an exhaustive assessment of problems that beset the simple conditional analysis. There is, for instance, a huge amount of literature on the "iffyness" of ability statements, which took its starting point with Austin's (1956) famous criticism of the simple conditional analysis, which I will not go into here. 2 This is consistent with Lewis's (1973) use of the term. 
of the United States, you would be more powerful than you are now; if birds didn't have feathers, they would be cold in the winter. Here are some false ones. If I had gotten up at 8, I would have heard the church bells; if you were the president of the United States, you would be less powerful than you are now; if birds didn't have feathers, they would wear little knit sweaters.

What makes counterfactuals true and false, respectively? The standard semantics (Lewis 1973; Stalnaker 1968) states the truth conditions of a counterfactual in terms of possible worlds. Possible worlds are complete ways things can be - "single, maximally inclusive, all-encompassing situation[s]" (Menzel 2016).

Theoretically, possible worlds can be thought of as a variety of things. Lewis famously thought of them as real entities, viz. entities of the same kind as the actual world, but causally, temporally, and spatially completely unrelated to it (Lewis 1986). Most philosophers, however, do not think of them as material entities. Usually, possible worlds are thought of as abstract objects (Plantinga 1974, 1976), as sets of propositions (Adams 1974; Fine 1977) or as "useful theoretical entities having no independent reality" (Menzies 2014) at all. I am not going to commit on this issue throughout this book; you are free to make your pick.

We can now introduce a very important notion: the notion of similarity, or closeness. Possible worlds resemble each other with respect to what is true in them. They resemble each other in certain aspects, but also overall. And this allows us to impose an ordering on them. This ordering can be thought of in spatial terms:

[I]magine all possible worlds spewed out across the logical universe, with the actual world in the center [and suppose that] the relative distance from a given world to the actual world is a measure of the relative similarity of that world to the actual world. (Heller 1999: 116).

The more similar a world is to the actual world, the closer it is to the actual world in modal space. The more dissimilar, the further away. This is all the apparatus we need to state the truth conditions for counterfactuals. Because we can now state, with Lewis (1973: 16), that

STANDARD-1. "If $\mathrm{p}$ were the case, $\mathrm{q}$ would be the case" is true at a world $\mathrm{w}$ if and only if (i) some $\mathrm{p}$-world where $\mathrm{q}$ holds is closer to $\mathrm{w}$ than any $\mathrm{p}$-world where $\mathrm{q}$ does not hold or (ii) there is no possible $\mathrm{p}$ world, in which case the counterfactual is vacuously true.

Counterfactuals with impossible antecedents need not concern us in this chapter, so let's ignore condition (ii). The important condition for our purposes here is condition (i): the condition that some $\mathrm{p}$-world where $\mathrm{q}$ holds is closer than any $\mathrm{p}$ world where q does not hold. Complicated as the condition seems at first sight, 
the idea underlying it is actually very simple and intuitive. It is just that "a counterfactual is true, if it takes less of a departure from actuality to make the antecedent true along with the consequent than to make the antecedent true without the consequent” (Menzies 2014).

Apply this to an example. Why wouldn't birds wear sweaters, if they didn't have feathers? The answer provided by STANDARD-1 is: because it is not the case that some world in which they lack feathers, but wear sweaters is closer than any world in which they lack feathers and don't wear sweaters. Many worlds in which they lack feathers and don't wear sweaters are much closer.

Despite the fact that (i) is quite intuitive on second glance, it is obviously a bit unwieldy. I will therefore help myself to a modification of the condition, which we gain if we accept what is known as the limit assumption: the assumption that there are always closest p-worlds. ${ }^{3}$ Plausible at first, the limit assumption is actually very controversial, and Lewis takes it to be false. Take a counterfactual with the antecedent "If I were taller". The problem with such a counterfactual is that it is hard to see what the closest worlds are in which I am taller. Since height is continuous, there will be an infinity of increasingly similar worlds between the most similar world in which I am exactly one centimeter taller and the actual world. Which is the most similar world in which I am taller (at all), then? It seems like there is none.

To deal with the possibility of ever more similar worlds, Lewis formulated the truth conditions of counterfactuals along the lines of STANDARD-1. Neglecting problems with the limit assumption, however, one can simplify the first condition quite a bit and restate the truth conditionals for counterfactuals as follows:

STANDARD-2. "If $p$ were the case, $q$ would be the case" is true at a world $w$ if and only if (i) the $\mathrm{p}$-worlds that are closest to $\mathrm{w}$ are $\mathrm{q}$-worlds or (ii) there is no possible $\mathrm{p}$ world, in which case the counterfactual is vacuously true. ${ }^{4}$

Instead of postulating that some world in which the antecedent and the consequent holds be closer to w than any world in which the antecedent is true, but the consequent false, our new condition (i) simply postulates that the closest worlds to $\mathrm{w}$ in which the antecedent is true are worlds in which the consequent is true as well. The new condition implies the earlier condition, given that there

3 One of the central differences between the otherwise very similar views of Lewis and Stalnaker on counterfactuals is that Stalnaker accepts, while Lewis denies the limit assumption (Lewis 1973: 78).

4 This comes very close to Stalnaker's version of the semantics. 
are closest antecedent-worlds, which is just what the limit assumption presupposes.

In what follows, I will act as though the limit assumption were unproblematic and use STANDARD-2 whenever I state or refer to the truth conditions for counterfactuals. Note, though, that I am thereby choosing simplicity over precision. I trust that the reader will bear in mind that problems with the limit assumption can be resolved by employing the more sophisticated STANDARD-1 instead.

So far, we have only looked at counterfactuals with false antecedents. Let us now briefly look at a less common, but equally important case for our concerns in this chapter: counterfactuals with true antecedents. Consider the counterfactual "If I had a dog, I would go for a walk every day", and suppose I actually have a dog. How do we determine the truth conditions of such a counterfactual? As before: move to the closest antecedent-worlds and determine whether the consequent is true in them. Which ones are the closest antecedent-worlds, though? Here, people diverge.

Most people accept what is called "centering": the idea that every world is closest to itself and no other world is as close. Given centering, the closest antecedent-worlds for counterfactuals with true antecedents will always be only the actual world itself. Thus, to find out whether it is true that I would go for a walk every day if I had a dog, all we need to check is whether I actually go for a walk every day. If I do, the counterfactual is true. If I don't, it's false.

But not everyone endorses this part of the semantics. Nozick (1981: 176), for instance, rejects it, and holds that the sphere of the closest antecedent worlds will contain a few non-actual worlds, even in the case of counterfactuals with true antecedents. Hence, to determine whether or not I would go for a walk every day, if I had a dog, it will not do to determine whether or not I go for a walk every day in the actual world. It will also have to be true that I go for a walk every day in the other closest, non-actual worlds in which I have a dog. The counterfactual is true only if I go for a walk every day in those worlds as well.

Whether or not centering is a good idea will not - and need not - be settled here, because it is irrelevant to the arguments that are going to be developed in what follows. For simplicity, I will assume that it holds. Whenever I anticipate that issues about centering could be thought relevant to one of my arguments, I'll provide a brief commentary on why the argument works either way.

This very brief introduction into the semantics of counterfactuals will do. A counterfactual (with a possible antecedent) is true if and only if the consequent is true in the closest antecedent worlds. What we need to do now is apply this to the simple conditional analysis. 
The simple conditional analysis states that an agent has an ability to $\phi$ if and only if the agent would $\phi$, if she intended to $\phi$. What this translates into, on the standard semantics, is that an agent has an ability if and only if the agent $\phi$ 's in the closest possible worlds in which the agent intends to $\phi$. Thus, to find out whether an agent has an ability, we need to move to the closest worlds in which the agent intends to $\phi$ and check whether she effectively $\phi$ 's in those worlds. If she does, she has the ability. If she doesn't, she lacks it.

Quite intuitive, at first glance. As we'll see in the next sections, though, the view is not very plausible at all, because it runs into problems with so-called "masks" (2.2) as well as cases of impeded intentions (2.3), and it fails to account for the distinction between general and specific abilities (2.4), degrees and the corresponding kind of context sensitivity of ability ascriptions (2.5), as well as for non-agentive abilities (2.6).

\subsection{Extensional inadequacy I: Masks}

In this and the next section, we will see that the conditional analysis is extensionally inadequate; the view invites counterexamples which show that the conditional analysis is neither necessary nor sufficient for an agent to have an ability. In this section, we will focus on the first type of counterexamples counterexamples which show that the conditional analysis is not necessary.

As I'll argue, then, it may well be that some agent has an ability to $\phi$, and yet it may be false that the agent would $\phi$ if she intended to $\phi$. How? Simply thus: something may interfere with the exercise of the ability. Call an ability which is had but whose exercise would be interfered with a masked ability, and call the interfering factor a mask (Johnston 1992). Here are some everyday examples of masked abilities. My ability to swim may be masked by a cramp. My ability to sing a certain song may be masked by my hoarseness or my shyness or my forgetfulness of the lyrics. My ability to hit the bull's eye may be masked by my sudden dizziness.

In all of those cases, the counterfactual is false: it is not true that I would swim or sing or hit the bull's eye if I intended to do so, because the closest intention worlds will be worlds in which the mask will be present. In the closest worlds in which I form the intention, I thus fail. Yet I do have the relevant abilities. Hence, masking cases show that the truth of the counterfactual is not necessary.

There is an even simpler way to come up with masking cases: all we need to do is cite cases in which an agent who has an ability to $\phi$ actually intends to $\phi$ and fails. Whenever an agent has an ability to $\phi$, but fails to realize her intention 
to $\phi$, we have a case of masking: something came in the way. Again, the counterfactual is false in such a case: it is not the case that the agent would $\phi$, if she intended to, because she did intend to $\phi$ and didn't $\phi$.

This is one of the places where centering may be thought to play a role in the argument. If centering is false, then the closest worlds contain not just the actual world, but also other worlds in which the agent intends to $\phi$. And couldn't it be that what masks the ability in the actual world is not present in those worlds? The smaller and more random the feature is which interferes with the exercise of the ability in the actual world, the more plausible it is that the same interference is not present in the other closest worlds, after all. Thus, one may think, it does not do to cite an actual intention and failure to falsify the counterfactual, if one rejects centering.

As far as I can see, this line of thinking is misled, though. Even if the closest worlds contain some in which the interfering factor is not present, this will not help to rescue the truth of the counterfactual. To see that, let's go through the truth conditions very carefully. First, let us assume that the limit assumption is true, and a counterfactual is therefore true if and only if all of the closest antecedent worlds are consequent worlds. In that case, the conditional analysis postulates that the agent $\phi$ 's in all of the closest worlds in which she intends to $\phi$. Let's now assume that centering is false. The closest intention worlds will now contain some non-actual worlds that are still very close. Let's also assume, for the sake of the argument, that the agent does in fact $\phi$ in some of those closest worlds.

The important point is that the counterfactual will still be false in that case. For as long as the actual world is among the closest intention worlds, a failure to $\phi$ in the actual world will suffice to falsify the counterfactual. Rejecting centering is to reject that the actual world is the sole member of the set of closest worlds. It is not to reject that the actual world is among the closest worlds. Whether or not we accept centering: the actual world will always be among the closest worlds to itself. Hence, the argument runs smoothly, no matter whether or not centering is accepted or not.

What if we also reject the limit assumption? Then we will have to work with the slightly more complicated truth condition for the counterfactual to be true. But even then, the counterfactual will be false, if the actual world is an antecedent, but not a consequent world.

Suppose the limit assumption is false. Then a counterfactual is true if and only if there is some antecedent world in which the consequent is true that is closer than any antecedent world in which the consequent is false. Thus spelled out, the simple conditional analysis postulates that there be some intention world in which the agent $\phi$ 's that is closer than any intention world in which 
the agent does not $\phi$. Let's again reject centering. The sphere of the closest intention worlds will be slightly larger than the sphere containing only the actual world. And let's assume, for the sake of argument, that the agent does in fact succeed to $\phi$ in some of the closest intention worlds.

Even so, the counterfactual will be false, given that the agent intends to $\phi$ and fails in the actual world. That is because it is not true, in that case, that some world in which the agent intends to $\phi$ and $\phi$ 's is closer than any world in which the agent intends to $\phi$ and fails. There may be closest worlds in which the agent intends to $\phi$ and succeeds, but none of them will be closer to the actual world than the actual world itself. They will be just as close, but not closer. No matter how we position ourselves regarding the various ways of analyzing counterfactuals, an actual intention and failure suffices to falsify the counterfactual figuring in the conditional analysis.

Masks provide a simple lesson, then. The counterfactual is too strong to be a necessary condition for an agent to have an ability. Abilities can be had, and yet it may be true that an intention would fail to be realized. Actual intentions do quite often fail to be realized. And if no actual intention is formed, the world may be such that it would fail to be realized. This is all we need to show that the simple conditional analysis fails to state a necessary condition for an agent to have an ability.

Can the conditional analysis be ameliorated? At first sight, it may seem like a good idea to add specifications into the antecedent to exclude masking cases. Aune suggests that the relevant conditional in the case of abilities is not "I will if I choose", but rather "if I want to lift it, try to lift it, and nothing interferes with my attempt, I will certainly succeed" (Aune 1963: 338, my emphasis). ${ }^{5}$ On closer inspection, though, this strategy proves problematic.

To see that, let's take a step back from abilities and look into the recent literature on dispositions. For readers familiar with that literature, masks and their impact on conditional analyses are not a dark horse. Conditional analyses have been very prominent not just in connection with abilities, but also, and in fact foremost, in connection with dispositions.

According to what we can call the simple conditional analysis of dispositions, something has a disposition to exhibit some behavior if and only if it would exhibit that behavior if it were to undergo an appropriate stimulus (Ryle 1949; Goodman 1954; Quine 1960). Sugar is soluble, on this view, if and only if it would dissolve if it were placed in liquid. A poison is toxic if and only if it

5 We also find the formulation: "if Jones were to try to do X under absolutely noninterfering, or nonfrustrating, conditions, he would be successful” (ibid.: 401). 
would cause harm if it were ingested. A person is irascible if and only if she would freak out if she were provoked.

The analogy between the conditional analysis of abilities and the conditional analysis of dispositions should be obvious. In both cases, a counterfactual tie is postulated between some trigger and the manifestation of the ability and the disposition, respectively. In view of this similarity, it does not come as a surprise that cases of masking have been raised as a problem against the conditional analysis of dispositions as well. A poison may be ingested together with an antidote, in which case it is not true that the poison would cause harm if it were ingested (Bird 1998). ${ }^{6}$ An irascible person may be provoked, but her wife may be around to soothe her. In that case, she would not freak out if she were provoked. And so forth.

Masking is a very prominent objection against the simple conditional analysis of dispositions and is extensively discussed in that connection. And of course, we find an analogue of Aune's proposed amelioration of the counterfactual in connection with dispositions, too. Most famously, the analogous suggestion is made by Lewis when he writes that

we might offhand define a poison as a substance that is disposed to cause death if ingested. But that is rough (...) [W] should really say 'if ingested without its antidote'. (Lewis 1997: 145)

The suggestion is clear. Just as Aune suggests for abilities, the idea is to retain the general scheme of the analysis, but to specify the antecedent in such a way that masks are explicitly ruled out. A number of authors (i.e. Choi 2006, 2008; Gundersen 2002; Mumford 1998, ch. 4) have followed Lewis's lead. While the accounts differ in their exact formulation, they all state essentially the same idea: $\mathrm{x}$ has a certain disposition to $\phi$ if and only if $\mathrm{x}$ would $\phi$ if a stimulus occurred and conditions $C$ obtained, where conditions $C$ will have to be understood to exclude masks. Following Whittle (2010), we can call this the specifier approach.

There is definitely something to this approach. It certainly calls attention to a certain discomfort I believe most people have when they first encounter the objection of masks. Somehow it seems clear that when we say things like "to be toxic is to cause harm, if ingested", we implicitly intend "if ingested" to be understood as something along the lines of "if ingested under the right conditions". Analogously for the conditional in "to have an ability to swim is to swim if one tried to swim". Of course, what is meant is "if one tried to swim under the right

6 Bird calls masks in general "antidotes". 
conditions". And if that's what is meant then coming up with masks is just cheating; it exploits the fact that we have not made that additional condition explicit right away. Once it is made explicit, however, those apparent counterexamples turn out to be simply irrelevant for the truth of the conditional.

Legitimate as this intuition seems, however, there are two problems with the specifier approach. The first is that it is far from clear whether $\mathrm{C}$ - the good conditions - can be spelled out in a non-trivial way at all. That is because for each disposition and ability, respectively, there is an indefinite variety of potential masks:

The kazoo's disposition to buzz when blown through (...) can be masked by stuffing the kazoo full of paper. It can also be masked by coating the kazoo's vibrating reed with wax; or by putting one's finger over the end of the kazoo; or by submerging the end of the kazoo in honey; or .... Given any finite list of potential maskers (...), it seems that a masking situation not on that list could always be imagined. (Fara 2005: 51)

Fara's point is clear. No specification of the circumstances could ever be complete when it comes to the exclusion of the crucial counterexamples. It thus seems that the only way of specifying $C$ would have to be the specification of a feature that all of the potential counterexamples have in common. Given the diversity of the examples, however, it is hard to think of such a feature.

Indeed, as Fara rightly points out, "the only relevant property that cases of masking have in common with each other is that they are cases of masking" (ibid.). And this shared property helps little when it comes to the specification of C. For surely, we should not say that something has a disposition to $\phi$ if and only if it would $\phi$ in the absence of masks. So formulated, the conditional states a triviality; it states that something has a disposition to $\phi$ if and only if it $\phi$ 's as long as nothing prevents it from doing so. Obviously, on this account, every object has every disposition whatsoever (Bird 1998: 231).

Abilities do not differ from dispositions when it comes to the diversity of potential masks: my ability to swim can be masked by my legs being bound together, by some weight on my feet, by panic, by a cramp, by too many people leaving too little space... But those masks do not seem to have anything in common besides the fact that they prevent my ability to swim from being exercised. Hence, the problem of the plentitude and diversity of potential counterexamples arises for abilities as well; it will either turn out impossible to specify $\mathrm{C}$ or we are once more heading towards the triviality problem.

Aune's suggestion falls prey to the second horn of the dilemma: stating that to have an ability is for it to be true that the agent would $\phi$, if she intended to and nothing interfered, does indeed seem trivial. For what else is an interference 
than simply a feature of the situation that results in a non-occurrence of whatever actions is intended from being performed?

The second problem with the specifier approach is related, but different. For even if the circumstances which pose an interference could be specified, the specifier view will run into problems. To see that, suppose you and I both have the ability to hit the bull's eye. We both hit it in seven out of ten cases. In three out of ten cases, the ability is masked. But now suppose those three cases are not the same ones in your case and in mine. Whenever you fail, I succeed. Whenever I fail, you succeed. We both have the ability to hit the bull's eye, but that ability is masked in completely different conditions.

This case poses a problem for the specifier approach, because the specifier approach does not provide any means to specify our shared ability to hit the bull's eye. Your ability will be the ability to hit the bull's eye in conditions in which the set of impediments A does not obtain, whereas my ability will be the ability to hit the bull's eye in conditions in which a different set of impediments B does not obtain. Even if the impediments could be specified in a nontrivial way, then, the specifier view fails to account for the fact that one and the same ability may be masked by completely different factors in different agents (cf. Manley and Wasserman 2008: 75n20).

We can conclude that masks are problematic for the conditional analysis: there is good reason to doubt that there is a non-trivial counterfactual, the truth of which will turn out to be necessary for the having of an ability.

\subsection{Extensional inadequacy II: Impeded intentions}

Masks show that the conditional analysis does not state a necessary condition for the having of an ability. Let me now argue that it also fails to state a sufficient condition. To see the problem, let's recall that an agent has the ability to $\phi$, according to the simple conditional analysis, if and only if she would $\phi$, if she intended to $\phi$. Why is that condition not sufficient? The reason is very easily stated: it may well be true that an agent would perform a certain action if she intended to perform it, but be unable to form the intention to begin with. In that case, the counterfactual is true, while the ability is lacking (Moore 1912, ch. 1 \& 7; Chisholm 1966, 1976; Lehrer 1968; van Inwagen 1983: 119; Whittle 2010).

Illustrations are easy to come by. Comatose patients (van Inwagen 1983: 119) lack the ability to raise their arms. Yet, the conditional analysis yields that they have the ability. That is because the counterfactual turns out true for the coma patient: if she intended to raise her arm, she would. To see that, let's frame the issue in the standard semantics for counterfactuals. To find out whether 
the coma patient has the ability to raise her arm, we have to move to the closest possible worlds in which she intends to raise it, according to the conditional analysis. But since the coma prevents the agent from forming an intention to raise her arm in the first place, we have to move beyond the coma worlds in order to reach the closest possible intention worlds. Since the agent is not in a coma in those worlds, however, there is no reason to assume that she would not succeed in raising her arm in those worlds. Hence, the counterfactual turns out true, while the agent obviously lacks the ability.

In case you wonder whether it makes a difference which motivational state figures in the counterfactual, let me point out that it does not. The coma case is a counterexample to any version of the conditional analysis. A coma prevents an agent from forming any motivational state of raising her hand whatsoever coma patients can neither try to raise their hands, nor can they intend, choose, or want to raise them. No matter what motivational state we insert, the analysis fails.

Can we think of further cases with such an impact? I think we can. Just consider a brainwashed follower of a cult. ${ }^{7}$ Can the person leave the cult? No. Being as brainwashed as she is, she cannot. But would she leave the cult, if she formed the motivation to leave? Presumably yes. What the brainwash plausibly does is prevent the agent from forming the motivation to leave to begin with. Hence, the closest motivation worlds will be worlds in which the agent is not brainwashed. And hence, there is nothing to prevent the agent from leaving the cult in those worlds.

Again, it seems that the counterexample works against all versions of the conditional analysis alike. Brainwash prevents the agent from forming any motivation to leave whatsoever. Intending, choosing, wanting, and trying to leave are all beyond the range of things a thoroughly brainwashed person can do. Hence, the example seems to refute the conditional analysis as such, and not just a particular version of it.

What all this shows is that the conditional analysis runs into deep structural problems with a certain class of cases. Whenever an agent cannot $\phi$ in virtue of an impairment that prevents her from forming the intention (or any kind of mo-

7 An example that is often cited is that of a phobic, who cannot touch spiders because she cannot form the intention or make the attempt to begin with (Lehrer 1968). I don’t find that example particularly well chosen, because it is not entirely clear whether the phobic is really impaired on the motivational level. Furthermore, the phobia case invites discussions about potential variances in plausibility between the various versions of the conditional analysis with different motivational states in the antecedent. For those reasons, I prefer to talk about coma patients and brainwashed agents instead. 
tivation) to $\phi$ to begin with, the counterfactual will come out true, while the agent obviously lacks the ability to $\phi$. Let's call this the problem of the impeded intention.

It is important to note that the problem of the impeded intention does not hinge on the claim that there is no good sense of "ability" in which the coma patient does in fact have the ability to raise her arm (and likewise for the brainwash case). Presumably, there is such a sense. The ability to raise one's arm may not be the best example to see that. But consider abilities which actually require a lot of expertise. Suppose the coma patient is a world-famous juggler. There is, I take it, good sense to be made of the claim that the coma patient still has the ability to juggle.

That this claim can be made perfect sense of does not attenuate the problem of the impeded intention, however. There may be a good sense in which the coma patient can juggle. Yet, there is an equally good sense in which she cannot juggle. The reason for the lack of that ability is obviously that the coma patient is in a coma. Obviously, a coma is an impediment to juggling, and a very severe one at that. Hence, a comatose agent is unable to juggle (in a very sense of "ability"). I don't think there is much room for disagreement here. The problem with the conditional analysis is that it fails to account for that very good sense in which the coma patient lacks various abilities.

The problem of the impeded intention is a very deep-running problem, then, because it shows that the whole structure of the counterfactual is ill-suited when it comes to analyzing abilities. The ability to $\phi$ depends, in part, on the ability to form the intention to $\phi$. And so, the mere fact that there is a counterfactual connection between the intention and the agent's performance is, by its very nature, insufficient to show that the agent has the ability. When the agent cannot form the intention to $\phi$ to begin with, she also cannot $\phi$. The simple conditional analysis seems to exhibit a deep structural flaw at this point.

There is a natural rejoinder for the proponent of the simple conditional analysis. If the problem of the impeded intention is that the agents in the examples cannot form the intention in the first place, why not simply add a second condition? Why not simply postulate that the agent also has to be able to form the intention in the first place? This further requirement, it can be argued, is already implicit in the conditional analysis, and once pressed, the proponent of the conditional analysis may simply state it as an additional condition of her view. According to the full account, then, there has to be a counterfactual connection between the agent's intentions and performances on the one hand, but the agent also has to be able to form the intention in the first place.

As we will see in connection to the account of abilities that I will lay out in chapter 4 , this strategy is actually a very good idea. The ability to $\phi$ does indeed 
require the agent also to have the ability to form the intention to $\phi$. The problem is just that it cannot be spelled out with the toolbox available to the proponent of the conditional analysis as it stands. For what is it to be able to form the intention to $\phi$ ? It is to have yet another ability. But surely, it will not do to simply reapply the conditional analysis to that ability all over: it is not a very promising move to suggest that the ability to intend to $\phi$ is had if and only if the agent would form the intention to $\phi$, if she intended to form the intention to $\phi .^{8}$ The analysis becomes either circular at this point or we slip into a regress. Either way, we will not have made much progress.

The problem remains. As the coma case and the brainwash case show, it may well be that an agent would $\phi$ if she intended to $\phi$, and yet she may be utterly unable to $\phi$. We can conclude that the simple conditional analysis, besides failing to state a necessary condition for an agent to have an ability, also fails to state a sufficient condition.

\subsection{The problem with general and specific abilities}

In the last two sections, we saw that the simple conditional analysis runs into extensional problems. In this and the next two sections, I'll show that the simple conditional analysis also fails to account for the other three adequacy conditions within a comprehensive view of abilities.

Let's start with the condition that any comprehensive view of abilities will have to elucidate the relation between general and specific abilities. As I will argue, the simple conditional analysis fails to account for both general and specific abilities individually. Consequently, the relation between the two remains in the dark as well.

That the simple conditional analysis fails to provide an account of general abilities is quite easy to see. Take the general ability to do a handstand. Obviously, you can have that ability, even if you are presently dizzy or on a shaky boat, say. That's just what makes the ability general: whether or not you have them does not hinge on the temporary features of your current situation; temporary unfavorable circumstances do not deprive you of your general abilities. The specific ability to do a handstand, in contrast, does hinge on such features. You cannot do a handstand here and now when you are here and now dizzy or on a shaky boat.

8 Moore (1912, ch. 7) makes this suggestion: having the ability to choose, he notes, is for it to be the case that the agent would choose, if she chose to choose. 
It should be quite obvious that the simple conditional analysis fails miserably as an account of general abilities. A counterfactual is true if and only if the closest worlds in which the antecedent is true are also worlds in which the consequent is true. To find out whether a counterfactual holds, that is, we move away from the actual world only far enough to make the antecedent true. To find out whether someone would do a handstand if she intended to, say, we move to the very closest worlds in which the agent intends to do a handstand. As much as possible remains as it is in actuality in those worlds. Hence, a dizzy agent is dizzy in those worlds, and an agent on a shaky boat is still on a shaky boat in those worlds.

Will the agent do a handstand in those intention worlds? Of course not. The counterfactual will come out false for dizzy agents and agents on shaky boats, then. But we said that the agent can have the ability to do a handstand despite her dizziness and unfavorable location, respectively. Hence, the counterfactual does not do justice to abilities that are general in that way.

Quite generally put, the problem with an account of general abilities in terms of the conditional analysis is that a general ability abstracts away from very many features of an actual situation, whereas the semantics of a counterfactual factors all of the features of the actual situations in when determining the closest antecedent worlds. Hence, the tension between general abilities and a counterfactual account of such abilities runs rather deep.

How about specific abilities? At first one may think that the conditional analysis is rather well suited as an account of abilities of that kind. Especially in view of the failures it exhibits with respect to general abilities, this may seem quite plausible at first. A specific ability is an ability to perform an act in a given situation. It thus seems like a good idea to look at what the agent would do in an intention situation that is as similar as possible to that very situation. Hence, it seems as though specific abilities do not conflict with the semantics of the counterfactual in the destructive way as general abilities. When I am dizzy or on a shaky boat, I cannot do a handstand here and now, with those impediments in place. When it comes to specific abilities, it therefore seems to be a good feature of the semantics of counterfactuals that we look for intention worlds that resemble the actual world as closely as possible with respect to as many features as possible - including the impediments to the exercise of the ability that are actually in place.

The plausibility of this line of thought hinges on a topic we touched on earlier: the maskability of abilities. To see that, note that the reason I cited for the inappropriateness of the conditional analysis for general abilities can be formulated in terms of their maskability. General abilities can obviously be masked. The actual world can contain impediments to the exercise of the ability without 
thereby depriving us of the ability altogether. Dizziness and being located on a shaky boat are masks for one's general ability to do a handstand, for example.

The important point is that it is in virtue of the maskability of general abilities that that such abilities cannot be accounted for in terms of a counterfactual. The general ability is there despite the masks. Yet, the mask prevents the exercise of the ability. But since the worlds relevant to the truth of the counterfactual are the closest intention worlds of the agent, the mask will usually be present in such worlds. Hence, the counterfactual will turn out false whenever the actual world contains a mask for an agent's general ability.

We can conclude from this that the simple conditional analysis runs into problems with any maskable ability whatsoever. So the question we need to answer in order to find out whether or not the conditional analysis is suitable as an account at least of specific abilities is whether or not specific abilities can be masked or not. If they cannot, the conditional analysis will very plausibly state at least a necessary condition for the having of such abilities. If they can be masked, in contrast, then the simple conditional analysis is no more plausible as an account of them than of general abilities.

The question whether or not specific abilities are maskable is not easily answered; philosophers seem to have very different ideas about this question (e.g. Fara 2008; Vihvelin 2004; Clarke 2009). What is indisputable, though, is that unless virtually every fact, no matter how random or small, of the actual situation matters for whether or not some agent has the specific ability to perform some act in that situation, masks will be perfectly possible. Unless virtually every fact counts, there can always be a feature of the actual world that would come in the way of the exercise of an ability.

Does every fact count for an agent's specific abilities? Let's test your intuitions. Suppose you intend to hit the bull's eye and you fail, even though you usually hit it and the situation was perfectly favorable to your hitting it. Does your failure in that situation show that you did not have the specific ability to hit your target? Only when you answer this in the affirmative should you subscribe to the idea that specific abilities cannot be masked. If, in on the other hand, you believe that there is at least one good sense of "specific ability" according to which you did have the specific ability in that situation, albeit one that you failed to exercise, you should instead commit to the idea that even specific abilities can be masked.

If the latter is true - if some specific abilities can, in fact, be masked - then the simple conditional analysis will not yield the proper account of specific abilities, either. There can always be some influence present in virtue of which it is not true that the agent would perform some action, if she intended to perform it. 
Maskable specific abilities do not differ from general abilities at all in this respect.

My own view on specific abilities, upon which I will elaborate in chapter 4.5, is that there is indeed a good sense of "specific ability" according to which specific abilities can be masked. I take it to be reasonable that there are more or less specific abilities among the ones we rightly think of as specific. In fact, I believe that the most fruitful picture of the distinction between general and specific abilities is a more or less scalar one, according to which there are more or less general and specific abilities, with maximally specific ones on one end of the scale and maximally general ones on the other. They differ with respect to the set of facts that matters for whether or not the agent has the ability. Thus, I believe that even fairly specific abilities can be masked, while the most specific ones are essentially unmaskable $(\rightarrow 4.5 \mathrm{~d})$.

If this is correct, then the conditional analysis will at best state a necessary condition only of the most specific abilities we can think of - abilities which are such that any failed attempt shows that the agent did not have the ability in the first place. Slightly less specific abilities, abilities for which lots of facts about the actual situation matter, but which still allow for masks, in contrast, will be as badly accommodated by the counterfactual as any general ability.

Moreover, we should be very clear on one thing. Despite the fact that the simple conditional analysis may actually state a necessary condition for some very, very specific abilities, it is nevertheless inappropriate as an analysis of abilities of that kind. The reason for that has to do with the problem of the impeded intention once more. The problem of the impeded intention is not limited to general or even fairly general abilities at all. Quite to the contrary. While there may be some sense in which even the coma patient retains the very general ability to raise her arm, there is obviously no sense in which the coma patient has the specific ability to raise her arm in her particular situation, coma and all. Hence, the problem of the impeded intention seems even more pressing with respect to specific abilities, and a forteriori particularly pressing with respect to the most specific ones among the already specific abilities.

\subsection{The problem with degrees and context sensitivity}

In the last section, we saw that the simple conditional analysis fails to provide an account of general and specific abilities. In this section, I'll argue that the view also fails to account for the third adequacy condition: it fails to offer an account of degrees and the corresponding kind of context sensitivity that attaches to ability ascriptions. 
Abilities, we said in chapter 1, come in degrees. Glenn Gould has the ability to play the piano to a higher degree than I do; my dog has the ability to smell to a higher degree than most of us; my grandmother sews better, my cousin drives better, my father draws better than I do. One meteorologist may be better at predicting the weather than another. Two archers may be varyingly good at hitting the target. We practice things to become better at them. Abilities can be improved. We admire people who have outstanding abilities in some field or another. In a nutshell: degrees of abilities are phenomena with which we are all most familiar.

Looking more closely at degrees of abilities, we saw that the degree of an agent's ability can be influenced on two dimensions. The degree of an ability depends, first, on the quality of the agent's performances, and secondly on the range of circumstances across which the agent is able to deliver those performances. We called those two dimensions achievement and reliability, respectively.

We also said that the gradable nature of abilities results in a specific kind of context sensitivity of ability ascriptions. The degree of an agent's ability to $\phi$ required for the agent to count as having the ability to $\phi$ simpliciter varies across contexts. A darts player who misses $99 \%$ of the time, but who manages to hit the bull's eye every $100^{\text {th }}$ time does not usually count as having the ability to hit it. There may be contexts in which we count her as having the ability, though. She has what it takes; it is within the range of what she can do. The context, we said, determines some threshold along the scale of degrees of abilities above which an agent counts as having the ability simpliciter. Once we have degrees, then, we also have the mechanism for the corresponding context sensitivity.

The problem with the conditional analysis is that it is structurally ill-suited to yield an account of degrees of abilities. That is not to say that it fails to account for all variances in the degrees of two agents' abilities whatsoever. The conditional analysis can account for the fact that my partner is a better driver than I am, for instance, because (ignoring the problem of masks) it will rightly yield that while my partner can drive, I cannot. If he intended to drive, he would succeed. If I intended to drive, I would fail - I usually stall the engine right away. Given that anyone who has an ability at all has that ability to a higher degree than anyone who completely lacks it, my partner will come out more able than I.

But apparently, that does not give us the full range of degrees we are striving for. It does not, for instance, yield that my partner is a better driver than his friend. The problem with my partner's friend is not that she cannot drive at all. The problem is rather that she is not particularly good at it. This difference between my partner and his friend cannot be captured in terms of the counter- 
factual, though. Both would drive if they intended to. Yet, one of them would drive perfectly fine, whereas the other one of them would drive crazily.

Now, you may want to respond that this is not as much of a problem as it seems at first sight. Here is a suggestion for how the proponent of the conditional analysis may account for the difference between my partner and his friend. In a first step, let's attach values to the quality of an agent's performance. My partner gets a 10 and his friend gets a 1, say. And now let's specify the quality of the agent's performance in the consequent. Then we get the following two conditionals: if my partner intended to drive, he would drive with quality 10. If his friend intended to drive, she would drive with quality 1 . We can now say that the degree of an agent's ability is higher the higher the value attached to the agent's performance in the consequent.

This seems like a reasonable suggestion, but unfortunately it does not take us very far, either. First of all, the problem of masks re-emerges with new force. Suppose my partner is drunk. Then he, too, would drive crazily, if he intended to drive. In such circumstances, the simple conditional analysis, as we have just constructed it, yields that he is not good at driving at all. Again, the problem seems to be that the counterfactual ties agents' abilities way too closely to the circumstances they are actually in. This is a problem for the account of degrees to the same extent as it is a problem for the simple conditional analysis quite generally.

The problem I have just pointed out does not have primarily to do with degrees, though - in fact, it is just the problem of masks in a slightly different guise. (What it shows, though, is that the problem of masks is getting more and more complicated the closer one looks at it.) What really strikes me as a problem specifically in connection with degrees is something else: the problem is that conditionalists cannot account for the dimension of reliability at all.

How are we to measure the respective degrees of two meteorologists' abilities to predict the weather correctly, for example? Let us call our two meteorologists Fred and Ted. Fred has developed an algorithm, by means of which he can predict 99 percent of all weather constellations correctly. Ted has developed a faulty algorithm - it goes wrong for 45 percent of all weather constellations. Clearly, Fred is better at predicting the weather. Nevertheless, the counterfactual will often come out true for both of them alike. For suppose we are in a situation in which the weather is be predicted correctly by both meteorologists alike. Then the counterfactual will come out true for both of them. Who is the better meteorologist? According to the conditional analysis, they are on a par.

The problem that degrees of abilities pose for the conditional analysis is thus again that the counterfactual is too closely tied to the agent's actual situation. To account for degrees and specifically for the dimension of reliability, we need a 
means to evaluate the agent's performances across various kinds of circumstances. The counterfactual, however, only ever evaluates the agent's performance in one set of circumstances: the circumstances in which the agent intends to $\phi$ and which are otherwise as similar as possible to the agent's actual circumstances.

\subsection{The problem with non-agentive abilities}

There is one adequacy condition left to discuss. Any comprehensive view of abilities will have to account for agentive as well as non-agentive abilities. It should be obvious that the simple conditional analysis does not look too well-equipped with respect to that condition either. The simple conditional analysis, with its focus on the counterfactual tie between intentions on the one hand and performances on the other, is tailor-made for agentive abilities - abilities to perform paradigmatic instances of intentional actions. In cases of that kind, it is quite appealing to think that the having of such an ability has something to do with the tie between the agent's intention to do $\phi$ and her effectively doing $\phi$. To have the ability to jump, it has to be true that you would jump, if you intended to jump. To have the ability to sing, it has to be true that you would sing, if you intended to sing. As I argued, the simple conditional analysis fails even here, but at least it looks very plausible at first.

In the case of non-agentive abilities, however, the simple conditional analysis looks highly suspicious even at the outset. Non-agentive abilities are abilities to be engaged in certain behaviors that are not actions. It is therefore crucial for non-agentive abilities that they can (or can only) be exercised without a foregoing intention on the agent's part. A view which determines whether or not an agent has an ability by looking at the tie between intentions and performances thus seems inadequate right from the beginning. ${ }^{9}$

Now, perhaps the proponent of the simple conditional analysis need not admit defeat right away. In defense of her view, she might say: Look, it may seem odd to analyze abilities like the ability to digest or to produce saliva in terms of a counterfactual tie between intentions and performances. But in fact the analysis yields the right results. That is because of course I would digest, if I intended to. All I would have to do is eat something. And of course I would produce saliva, if I intended to. All I would have to do is bite my tongue.

9 Note that, again, nothing hinges on the particular motivational state that figures in the analysis. I will not comment on this any more as I go along. 
Thus, the counterfactual comes out true for those kinds of abilities. That is all that counts. ${ }^{10}$

There are at least two reasons why this strategy is unsuccessful. First, it yields an ugly disconnection between the conditions for having an ability and the conditions for exercising an ability. ${ }^{11}$ It seems very natural for the proponent of the conditional analysis to pair her claim that an ability to $\phi$ is had if and only if the agent would $\phi$, if she intended to, with the further claim that an ability is exercised if and only if the agent's $\phi$-ing results (non-deviantly) from an intention to $\phi$. In the case of agentive abilities, this seems very plausible. If I hit the bull's eye upon intending to hit it, I have exercised my ability to hit it. If I hit it by accident, though I actually intended to hit the outer ring, say, I take it that I have not exercised the ability to hit the bull's eye. My hitting the bull's eye is a mere accident in that case. The simple conditional analysis has a good story to tell about this: an ability is exercised if and only if the action follows an intention to perform it.

In the case of non-agentive abilities, things look rather different. Obviously, not every instance of digesting is a result of intending to digest. Indeed, the intended instances of digestion are very rare; usually, the digestion occurs without any foregoing intention on the agent's part. Yet, one will surely want to say that the unintended instances of digesting are, in fact, exercises of the agent's ability to digest. But here, the proponent of the conditional analysis does not have a good story; and whatever she says here will either result in a disanalogy with the agentive case or - if both cases are treated along the same lines - with the consequence that my hitting the bull's eye by accident counts as an exercise of my ability to hit the bull's eye as well. That does not seem auspicious.

Even if the proponent of the simple conditional analysis is willing to go for one of those options, however, there is a second and even stronger reason why non-agentive abilities cannot be accounted for by in terms of a counterfactual link between intentions and performances: there are some non-agentive abilities to $\phi$ which are never preceded by an intention to perform $\phi$. Just think of the examples I cited in chapter 2.6 - the ability to unintentionally read street signs, for instance. Or the ability to hear what someone says without listening. With respect to those examples, the limitations of the simple conditional analysis jump right at us. Clearly, it is not the case that I would read street signs unintentionally, if I intended to. Once I intend to unintentionally read street signs, I am bound to fail.

10 Erasmus Mayr (personal conversation) suggested this strategy to me, without, I think, endorsing it.

11 For the same point in connection with dispositions, see Vetter (ms.). 
Thus, the simple conditional analysis yields that I lack the ability to unintentionally read street signs. This is the wrong result.

What seems rather strange even in the case of digesting and producing saliva is therefore obviously wrong when it comes to other non-agentive abilities. We can conclude that abilities of the non-agentive kind are not properly accounted for by the simple conditional analysis. The modal tie between intentions and performances, while very plausible for agentive abilities, is obviously ill-suited to account for abilities of the non-agentive variety.

As we will see in chapter 4, my own view bears some similarities to the simple conditional analysis in that it, too, analyzes agentive abilities in terms of a modal tie between intentions and performances. I want to emphasize very clearly, however, that it does not stop there. For as I'll show in chapter 5, the view can be neatly extended to cover non-agentive abilities as well. However, the extension I suggest for my own view could, in principle, just as well be integrated into a framework operating with counterfactual conditionals. I'll briefly get back to all this in chapter 6.2, when I discuss the sophisticated conditional analysis I have been talking about earlier.

Since we are so far dealing with the conditional analysis as it is, however, let us just note that, as it stands, the view fails to provide the tools that would be needed to account not just for agentive, but also for non-agentive abilities. Together with the problems we noted in the last four sections, we therefore get the result that the simple conditional analysis runs into trouble with all of the adequacy conditions we have identified for a comprehensive view of abilities. Hence, the simple conditional analysis is certainly not the way to go.

\subsection{Upshot}

Let me once again walk you through the main insights of this chapter. In this chapter, we looked at what I called the simple conditional analysis. I used that notion as an umbrella term for any view which analyzes abilities in terms of a counterfactual tie between some motivational state to $\phi$ and the agent's effectively $\phi$-ing. For plasticity, I focused on one specific version of the view, namely that an agent has an ability if and only if she would $\phi$, if she intended to $\phi$.

Five problems were laid out for the simple conditional analysis, all of which consisted in failures to do justice to one of the adequacy conditions for a comprehensive view of abilities.

First, the simple conditional analysis fails to state a necessary condition, which was shown by pointing to cases of masking $(\rightarrow 2.2)$. In masking cases, there is some impediment which would prevent the agent from exercising her 
ability upon intending to do so. A cramp, for instance, masks the ability to swim. I argued that masks are a rather severe problem for the simple conditional analysis, because they show that the ability can be had, while the counterfactual turns out false. I also argued that the so-called specifier approach - an amelioration of the view that tries to exclude masks by specifying the antecedent - is much harder to spell out than one might think.

Secondly, the simple conditional analysis also fails to spell out a sufficient condition. This was established by showing that it may well be true that the agent would $\phi$, if she intended to $\phi$, and yet the agent may be unable to $\phi$, because she may be unable to form the intention to $\phi$ to begin with. I called this "the problem of the impeded intention" $(\rightarrow 2.3)$. Masks and impeded intentions show that the simple conditional analysis is extensionally inadequate.

Thirdly, the simple conditional analysis runs into problems with general and specific abilities $(\rightarrow 2.4)$. General abilities elude the analysis completely, because it is a characteristic feature of general abilities that they can be had, even if the agent is currently in unfavorable circumstances. Since the counterfactual is always evaluated at the closest intention worlds, the counterfactual will come out false in such cases. That is a problem.

The view works better with respect to specific abilities, although a lot hinges on the highly controversial question of whether or not specific abilities can be masked. Even if they cannot be masked, however, the simple conditional analysis will at best state a necessary condition for the having of such an ability, since the problem of the impeded intention is particularly powerful when it comes to specific abilities.

Fourthly, the simple conditional analysis fails to account for degrees of abilities and the corresponding kind of context sensitivity that attaches to ability statements $(\rightarrow 2.5)$. The degree of an ability to $\phi$ has to do, in part, with the range of possible circumstances across which the agent is successful in $\phi$-ing. Since the counterfactual only ever evaluates the closest circumstances in which the agent intends to $\phi$, however, the simple conditional analysis does not provide the tools to account for this fact. Consequently, the corresponding kind of context sensitivity of ability statements - one that has to do with a variable threshold on the scale of degrees - remains in the dark as well.

Finally, the simple conditional analysis fails to account for non-agentive abilities $(\rightarrow$ 2.6). The tie between intentions and performances, while initially plausible in the case of agentive abilities, will certainly not do the job when it comes abilities like smelling, digesting, or reading street signs without intending to read them. For abilities of that kind, the counterfactual will at best miss the point, and at worst come out straightforwardly false. 
Taken together, these problems indicate very clearly, I should think, that the simple conditional analysis is on the wrong track. That is not to say that it may not serve as a starting point for a much more sophisticated view operating with counterfactual conditionals. But as it stands, it is definitely mistaken. 